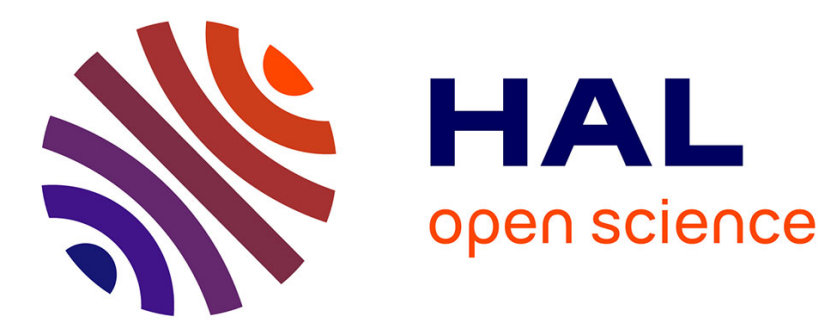

\title{
Cr2Te4O11: une structure à anions complexes (Cr2O10)14-
}

\author{
Georges Meunier, Bernard Frit, Jean Galy
}

\section{To cite this version:}

Georges Meunier, Bernard Frit, Jean Galy. Cr2Te4O11: une structure à anions complexes (Cr2O10)14-. Acta crystallographica Section B: Structural crystallography and crystal chemistry, 1976, 32 (1), pp.175-180. 10.1107/S0567740876002549 . hal-00156286

\section{HAL Id: hal-00156286 \\ https://hal.science/hal-00156286}

Submitted on 20 Jun 2007

HAL is a multi-disciplinary open access archive for the deposit and dissemination of scientific research documents, whether they are published or not. The documents may come from teaching and research institutions in France or abroad, or from public or private research centers.
L'archive ouverte pluridisciplinaire HAL, est destinée au dépôt et à la diffusion de documents scientifiques de niveau recherche, publiés ou non, émanant des établissements d'enseignement et de recherche français ou étrangers, des laboratoires publics ou privés. 
Acta Crystallographica Section B

Structural

Science

ISSN 0108-7681

Editor: Carolyn P. Brock

\title{
$\mathrm{Cr}_{2} \mathrm{Te}_{4} \mathrm{O}_{11}:$ une structure à anions complexes $\left(\mathrm{Cr}_{2} \mathrm{O}_{10}\right)^{14-}$
}

\author{
G. Meunier, B. Frit and J. Galy
}

Copyright $($ International Union of Crystallography

Author(s) of this paper may load this reprint on their own web site provided that this cover page is retained. Republication of this article or its storage in electronic databases or the like is not permitted without prior permission in writing from the IUCr. 
Acta Cryst. (1976). B32, 175

\title{
$\mathrm{Cr}_{2} \mathrm{Te}_{4} \mathrm{O}_{11}$ : une Structure à Anions Complexes $\left(\mathrm{Cr}_{2} \mathrm{O}_{10}\right)^{14-}$
}

\author{
Par Georges Meunier \\ Laboratoire de Chimie du Solide du CNRS, Université de Bordeaux I, 351 cours de la Libération, \\ 33405 Talence, France \\ BERNAND FRIT \\ Laboratoire de Chimie Structurale, U.E.R. des Sciences, 123, rue Albert Thomas, 87100 Limoges, France \\ ET JEAN GALY \\ Laboratoire de Chimie de Coordination, B.P. 4142, 31030 Toulouse Cedex, France
}

(Reçu le 12 mai 1975, accepté le 30 mai 1975)

\begin{abstract}
The crystal structure of $\mathrm{Cr}_{2} \mathrm{Te}_{4} \mathrm{O}_{11}$ has been determined from three-dimensional single-crystal X-ray data. Two $\mathrm{Cr}_{2} \mathrm{Te}_{4} \mathrm{O}_{11}$ units crystallize in a monoclinic unit cell of space group $P 2_{1} / c$ and dimensions $a=7.016, b=7.545, c=9.728 \AA$, and $\beta=99.69^{\circ}$. The structure is characterized by $\left(\mathrm{Cr}_{2} \mathrm{O}_{10}\right)^{14-}$ anions, formed by two octahedra sharing an edge and such units are connected to tellurium ( + IV) atoms and $\left(\mathrm{Te}_{2} \mathrm{O}\right)^{6+}$ groups. Tellurium $(+\mathrm{IV})$ atoms with their stereochemically active lone pairs have oxygen coordination [3] [Te(1)] and [3+1] [Te(2)]. $\mathrm{Al}_{2} \mathrm{Te}_{4} \mathrm{O}_{11}$ is isostructural with $\mathrm{Cr}_{2} \mathrm{Te}_{4} \mathrm{O}_{11}$. These results cor rect the compositions previously given for two phases reported in the $\mathrm{Al}_{2} \mathrm{O}_{3}$-and $\mathrm{Cr}_{2} \mathrm{O}_{3}-\mathrm{TeO}_{2}$ systems.
\end{abstract}

Dans un mémoire antérieur Frit \& Hagenmuller (1970)

étudiant les systèmes binaires $\mathrm{M}_{2} \mathrm{O}_{3}-\mathrm{TeO}_{2}(\mathrm{M}=\mathrm{Al}$, $\mathrm{Cr}, \mathrm{In})$ ont signalé l'existence de trois tellurites inédits de formule $\mathrm{M}_{2} \mathrm{Te}_{3} \mathrm{O}_{9}$. Les solides obtenus après fusion des composés $\mathrm{Al}_{2} \mathrm{Te}_{3} \mathrm{O}_{9}$ et $\mathrm{Cr}_{2} \mathrm{Te}_{3} \mathrm{O}_{9}$ étant vitreux, aucun cristal permettant une étude structurale fine n'avait pu être isolé; la similitude du spectre $X$ de poudre de ces deux phases et leur miscibilité en phase solide à $600^{\circ} \mathrm{C}$ indiquaient cependant un isomorphisme très probable. Le composé $\mathrm{In}_{2} \mathrm{Te}_{3} \mathrm{O}_{9}$, de structure différente, cristallise dans le système orthorhombique. Ce dernier résultat devait être confirmé ultérieurement par Redman, Binnie \& Mallio (1971).

Dans le cadre de nos études sur les composés oxygénés du tellure $(+I V)$ (Meunier, 1974; Meunier \& Galy, 1971, 1973; Galy \& Meunier, 1971; Meunier,
Darriet \& Galy, 1972, 1973; Galy, Meunier, Andersson \& Åström, 1975) la détermination de la structure cristalline d'un composé tel que $\mathrm{Cr}_{2} \mathrm{Te}_{3} \mathrm{O}_{9}$, comportant un élément de transition au degré d'oxydation + III, s'avérait importante, notamment pour l'établissement d'une classification structurale.

\section{Obtention de monocristaux - étude radiocristallographique}

Des monocristaux bien formés de la phase contenant le chrome ont été obtenus par une méthode de transport. L'agent de transport est le tétrachlorure de tellure $\mathrm{TeCl}_{4}$.

Un tube en vycor contenant $500 \mathrm{mg}$ environ d'un mélange intime de sesquioxyde de chrome et de bi- 
oxyde de tellure dans les proportions $\mathrm{Cr}_{2} \mathrm{O}_{3}-3 \mathrm{TeO}_{2}$, du tétrachlorure $\mathrm{TeCl}_{4}$ (à raison de $10 \mathrm{mg}$ par $\mathrm{cm}^{3}$ ) est scellé sous vide, puis introduit dans un four tubulaire maintenu à la température constante de $700^{\circ} \mathrm{C}$. Au bout de trois à quatre jours on observe la formation de petits cristaux prismatiques, de couleur verte, sur la paroi de la zone la moins chaude du tube.

Quelques monocristaux de la phase contenant l'aluminium ont été obtenus par chauffage à $650^{\circ} \mathrm{C}$ pendant huit jours suivi d'un refroidissement lent $\left(2{ }^{\circ} \mathrm{C}^{-1}\right)$ d'un mélange $\mathrm{Al}_{2} \mathrm{O}_{3}-4 \mathrm{TeO}_{2}$.

Une étude par diffraction $\mathrm{X}$, effectuée à l'aide de la radiation $K \alpha$ du cuivre a permis de montrer que ces deux phases cristallisaient dans le système monoclinique avec le groupe spatial $P 2_{1} / c$ et des paramètres cristallins voisins (Tableau 1). A l'aide de ces données et en respectant les conditions d'existence pour les diverses réflexions $h k l$ du groupe $P 2_{1} / c$, nous indexons parfaitement bien les spectres $X$ des poudres résultant du broyage des quelques cristaux isolés. Ces spectres correspondent bien à ceux antérieurement publiés. Ils sont portés au Tableau 2.

Tableau 1. Données cristallographiques de $\mathrm{Cr}_{2} \mathrm{Te}_{4} \mathrm{O}_{11}$

Phases
Symétrie
Paramètres
Groupe spatial
$D_{m}$
$D_{c}$
$Z$
et $\mathrm{Al}_{2} \mathrm{Te}_{4} \mathrm{O}_{11}$

\section{Structure cristalline - composition du monocristal (cas du chrome)}

Un monocristal prismatique de section quasi hexagonale (hauteur: $0,03 \mathrm{~mm}$, section: $0,020 \mathrm{~mm}$ ) a été sélectionné et monté sur un diffractomètre automatique Nonius CAD-3. Le rayonnement $\mathrm{X}$ utilisé était la radiation $K \alpha$ du molybdène sélectionnée à l'aide d'un monochromateur à lame de graphite. La technique de mesure consistait en un balayage selon $\omega$, l'angle de diffraction maximum pour la collection des données étant $\theta=45^{\circ}$. Seules les réflexions $(h k l)$ d'intensité $I>1,5 \sigma(\mathrm{I})$ ont été retenues, soit au total 970 réflexions. Les facteurs de diffusion des atomes de chrome, de tellure et d'oxygène proviennent des tables de Cromer \& Waber (1965). La seule correction effectuée a été celle par le facteur de Lorentz-polarisation. L'ensemble des calculs a été réalisé à l'aide d'un ordinateur Iris 80 en utilisant les programmes mis au point par $\mathrm{M}$. Saux.

La structure a été déterminée par déconvolution de la fonction de Patterson tridimensionnelle $P(u v w)$, par synthèse de Fourier et calculs de fonctions différences successives.
Tableau 2. Indexation des spectres $X$ de poudre de $\mathrm{Cr}_{2} \mathrm{Te}_{4} \mathrm{O}_{11}$ et $\mathrm{Al}_{2} \mathrm{Te}_{4} \mathrm{O}_{11}(\mathrm{Cu} K \alpha)$.

\begin{tabular}{|c|c|c|c|c|c|c|c|}
\hline & $\mathrm{Cr}_{2} \mathrm{~T}$ & ${ }_{4} \mathrm{O}_{11}$ & & & $\mathrm{Al}_{2}$ & $O_{11}$ & \\
\hline$h k l$ & $\begin{array}{c}d_{\text {calc }} \\
(\AA)\end{array}$ & $\begin{array}{l}d_{\text {obs }} \\
(\AA)\end{array}$ & $I / I_{o}$ & $h k l$ & $\begin{array}{c}d_{\text {catc }} \\
(\AA)\end{array}$ & $\begin{array}{c}d_{\mathrm{obs}} \\
(\AA)\end{array}$ & $I / I_{o}$ \\
\hline 100 & $\begin{array}{l}6,92 \\
5,93\end{array}$ & $\begin{array}{l}6,94 \\
5,94\end{array}$ & $\begin{array}{l}3 \\
4\end{array}$ & $\begin{array}{lll}0 & 0 & 2 \\
1 & 1 & 1\end{array}$ & $\begin{array}{l}4,70 \\
468\end{array}$ & 4,67 & 8 \\
\hline$\frac{1}{2}$ & & & & $\begin{array}{lll}1 & 1 & 1 \\
1 & 1 & 1\end{array}$ & $\begin{array}{l}4,68 \\
4,23\end{array}$ & 4,22 & 25 \\
\hline & 4,29 & 4,29 & 30 & & 3,66 & 3,68 & 90 \\
\hline $1 \frac{2}{2}$ & 3,731 & 3,732 & 100 & 102 & 3,61 & 3,59 & 70 \\
\hline $\begin{array}{ll}02 \\
0\end{array}$ & 3,662 & 3,660 & 76 & $0 ?$ & 3,47 & 3,47 & 80 \\
\hline 21 & 3,511 & 3,514 & 88 & 200 & 3,41 & 3,41 & 16 \\
\hline $\begin{array}{ll}0 & 0\end{array}$ & 3,458 & 3,458 & 15 & 12 & 3,28 & 3,27 & 2 \\
\hline 20 & 3,312 & 3,314 & 6 & 12 & 3,17 & 3,18 & 19 \\
\hline $2 \overline{1}$ & 3,211 & 3,213 & 24 & 21 & 3,10 & 3.09 & 100 \\
\hline 10 & 3,144 & 3,145 & 82 & 211 & 3,08 & 3,09 & 100 \\
\hline 1 & 3,133 & 3,134 & 60 & $\begin{array}{lll}12 & 1\end{array}$ & 3,02 & 3,01 & 46 \\
\hline 0 & 3,060 & 3,060 & 50 & 0 & 2,89 & 2,89 & 11 \\
\hline 21 & 3,055 & & & 1 & 2,824 & 2,828 & 22 \\
\hline 13 & 2,943 & 2,943 & 15 & 1 & 2,816 & 2,809 & 10 \\
\hline $1 \overline{3}$ & 2,873 & 2,871 & 30 & & 2,596 & 2,587 & 4 \\
\hline 11 & 2,860 & 2,862 & 30 & 20 & 2,518 & 2,517 & 3 \\
\hline & 2,835 & 2,835 & 8 & $\begin{array}{lll}0 & 23\end{array}$ & 2,402 & 2,401 & 8 \\
\hline $2 \overline{2}$ & 2,834 & 2,035 & 8 & $\begin{array}{lll}0 & 0 & 4\end{array}$ & 2,351 & 2,350 & 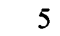 \\
\hline 2 & 2,628 & 2,628 & 8 & $3 \overline{1}$ & 2,301 & 2,301 & 5 \\
\hline 1 & $\begin{array}{l}2,569 \\
2439\end{array}$ & 2,569 & 6 & & & 2,241 & 30 \\
\hline $1 \frac{5}{3}$ & 2,435 & 2,433 & 12 & 3 & 2,193 & 2,193 & 10 \\
\hline & 2,433 & & & & 2,1 & 2,173 & 8 \\
\hline $2 \overline{3}$ & 2,399 & & & & & 2 & \\
\hline 0 & 2,397 & 2303 & 6 & & 2,117 & 2,114 & 4 \\
\hline & $\begin{array}{l}2,393 \\
2,391\end{array}$ & 2,390 & 0 & & 2,100 & 2,103 & 14 \\
\hline & 2,376 & 2,376 & 2 & & 2,039 & 2,040 & 9 \\
\hline & 2,326 & 2,325 & 10 & & 2,022 & 2,027 & 3 \\
\hline 1 & 2,285 & 2.284 & 28 & & 2,006 & 2,006 & 15 \\
\hline & 2,281 & & & 0 & 1,951 & 1,949 & $?$ \\
\hline & $\begin{array}{r}2,229 \\
\end{array}$ & & & 3 & 1,930 & & \\
\hline & $\begin{array}{l}2,228 \\
2,227\end{array}$ & 2,227 & 10 & & $\begin{array}{l}1,927 \\
1,927\end{array}$ & 1,929 & 9 \\
\hline & 2,213 & 2,214 & 8 & 04 & 1,8 & & \\
\hline & 2,170 & 2,171 & 10 & & & 1,866 & 12 \\
\hline & & 2,143 & 6 & & 1,85 & 1,853 & 10 \\
\hline & & 2137 & 12 & & & & 12 \\
\hline & 13 & & & 15 & 1,824 & 1,823 & 2 \\
\hline
\end{tabular}

L'examen des positions générales et particulières qu'implique le groupe $P 2_{1} / c$ fait ressortir cinq types de sites cristallographiques différents:

- un site à quatre équivalents:

$4(e)$ de coordonnées $x, y, z$

- quatre sites à deux équivalents:

$\begin{array}{lr}2(a) \text { de coordonnées } & 0,0,0 \\ 2(b) & \frac{1}{2}, 0,0 \\ 2(c) & 0, \frac{1}{2}, 0 \\ 2(d) & \frac{1}{2}, \frac{1}{2}, 0\end{array}$

Dans une première étape, la fonction de Patterson permet de situer deux atomes métalliques: l'atome de chrome, et un atome de tellure [Te(1)] dans des positions $4(e)$. Un calcul des facteurs de structure indique un accord relativement favorable avec les valeurs expérimentales, puisqu'il conduit à une valeur du facteur $R$ de 0,27 . Compte tenu de la formule chimique proposée $\left(\mathrm{Cr}_{2} \mathrm{Te}_{3} \mathrm{O}_{9}\right)$ cette solution permettait de situer la 
totalité des atomes de chrome et quatre des six atomes de tellure qu'implique la présence de deux motifs $\mathrm{Cr}_{2} \mathrm{Te}_{3} \mathrm{O}_{9}$ par maille. Dans ces conditions, les deux atomes de tellure restant ne pouvaient logiquement occuper que l'un des sites $2(a), 2(b), 2(c)$ ou $2(d)$. Les calculs effectués pour chacune de ces quatre hypothèses conduisaient malheureusement à une valeur de $R$ supérieure à 0,30 .

Nous avons donc entrepris une étude minutieuse de la fonction de Patterson. Celle-ci devait révéler l'existence d'un système de vecteurs impliquant la présence d'un atome lourd en position 4(e). Un calcul a donc été effectué en ajoutant un atome de tellure en position $\mathrm{Te}(2)$. Le facteur $R$ s'abaisse brutalement jusqu'à la valeur $R=0,16$. Une fonction différence a permis alors de localiser les atomes d'oxygène $\mathrm{O}(1), \mathrm{O}(2), \mathrm{O}(3)$, $\mathrm{O}(4)$ et $\mathrm{O}(5)$. Après quelques cycles d'affinement le facteur $R$ se stabilise au voisinage de la valeur 0,10 . Une nouvelle fonction différence calculée en tenant compte de ces cinq atomes d'oxygène laisse apparaître, outre une densité électronique sur le site $2(d)$, caractéristique de la présence d'un sixième atome d'oxygène en position indépendante $[\mathrm{O}(6)]$, les anomalies caractéristiques d'une agitation thermique anisotrope autour de la position des atomes lourds. Compte tenu de ces dernières observations un affinement faisant intervenir la matrice complète de l'algorithme a été effectué. Il conduit à la valeur finale $R=0,034$.

Les coordonnées réduites des divers atomes ainsi que leurs facteurs d'agitation anisotrope sont portés au Tableau 3, les distances et angles interatomiques au Tableau 4.*

Cette analyse structurale approfondie conduisant pour la composition du monocristal étudié à une formulation, $\mathrm{Cr}_{2} \mathrm{Te}_{4} \mathrm{O}_{11}$, différente de celle proposée, $\mathrm{Cr}_{2} \mathrm{Te}_{3} \mathrm{O}_{9}$, il nous a semblé nécessaire de reprendre l'étude chimique aussi bien du système $\mathrm{Cr}_{2} \mathrm{O}_{3}-\mathrm{TeO}_{2}$ que du système homologue $\mathrm{Al}_{2} \mathrm{O}_{3}-\mathrm{TeO}_{2}$ pour des compositions comprises entre $\mathrm{M}_{2} \mathrm{O}_{3} .3 \mathrm{TeO}_{2}$ et $\mathrm{M}_{2} \mathrm{O}_{3}$. $4 \mathrm{TeO}_{2}$ $(\mathrm{M}=\mathrm{Cr}, \mathrm{Al})$.

* La liste des facteurs de structure a été déposée au dépôt d'archives de la British Library Lending Division (Supplementary Publication No. SUP 31176 (7 pp.). On peut en obtenir des copies en s'adressant à: The Executive Secretary, International Union of Crystallography, 13 White Friars, Chester CHI 1NZ, Angleterre.
Tableau 4. Distances $(\AA)$ et angles $\left({ }^{\circ}\right)$ interatomiques Les écarts types sont indiqués entre parenthèses.

$\begin{array}{lclr}\mathrm{Te}(1)-\mathrm{Te}(2) & 4,058(1) & \mathrm{O}(1)-\mathrm{O}(4) & 2,88(1) \\ \mathrm{Te}(1)-\mathrm{Cr} & 3,481(2) & \mathrm{O}(1)-\mathrm{O}(5) & 2,75(1) \\ \mathrm{Te}(2)-\mathrm{Cr} & 3,358(2) & \mathrm{O}(1)-\mathrm{O}(21) & 2,93(1) \\ \mathrm{Cr}-\mathrm{Cr} & 3,101(3) & \mathrm{O}(2)-\mathrm{O}(21) & 2,54(1) \\ \mathrm{Te}(1)-\mathrm{O}(5) & 1,856(9) & \mathrm{O}(2)-\mathrm{O}(5) & 3,13(1) \\ \mathrm{Te}(1)-\mathrm{O}(11) & 1,872(9) & \mathrm{O}(2)-\mathrm{O}(6) & 2,80(1) \\ \mathrm{Te}(1)-\mathrm{O}(41) & 1,881(8) & \mathrm{O}(2)-\mathrm{O}(31) & 2,71(1) \\ \mathrm{Te}(2)-\mathrm{O}(2) & 1,891(10) & \mathrm{O}(3)-\mathrm{O}(2) & 2,73(1) \\ \mathrm{Te}(2)-\mathrm{O}(6) & 1,885(10) & \mathrm{O}(3)-\mathrm{O}(4) & 2,68(1) \\ \mathrm{Te}(2)-\mathrm{O}(31) & 1,846(9) & \mathrm{O}(3)-\mathrm{O}(5) & 2,62(1) \\ \mathrm{Te}(2)-\mathrm{O}(51) & 2,562(10) & \mathrm{O}(3)-\mathrm{O}(21) & 2,83(1) \\ \mathrm{Cr}-\mathrm{O}(1) & 1,970(9) & \mathrm{O}(4)-\mathrm{O}(5) & 2,76(1) \\ \mathrm{Cr}-\mathrm{O}(2) & 2,020(10) & \mathrm{O}(4)-\mathrm{O}(21) & 2,72(1) \\ \mathrm{Cr}-\mathrm{O}(3) & 1,957(9) & \mathrm{O}(5)-\mathrm{O}(11) & 2,76(1) \\ \mathrm{Cr}-\mathrm{O}(4) & 1,951(9) & \mathrm{O}(5)-\mathrm{O}(41) & 2,71(1) \\ \mathrm{Cr}-\mathrm{O}(5) & 1,960(9) & \mathrm{O}(6)-\mathrm{O}(31) & 2,76(1) \\ \mathrm{Cr}-\mathrm{O}(21) & 1,987(10) & \mathrm{O}(11)-\mathrm{O}(41) & 2,82(1) \\ \mathrm{O}(1)-\mathrm{O}(2) & 2,92(1) & & \\ \mathrm{O}(5)-\mathrm{Te}(1)-\mathrm{O}(11) & 95,5(4) & \mathrm{O}(5)-\mathrm{Cr}-\mathrm{O}(2) & 103,6(4) \\ \mathrm{O}(5)-\mathrm{Te}(1)-\mathrm{O}(41) & 93,0(4) & \mathrm{O}(2)-\mathrm{Cr}-\mathrm{O}(21) & 78,8(4) \\ \mathrm{O}(11)-\mathrm{Te}(1)-\mathrm{O}(41) & 97,4(4) & \mathrm{O}(1)-\mathrm{Cr}-\mathrm{O}(4) & 94,5(4) \\ \mathrm{O}(2)-\mathrm{Te}(2)-\mathrm{O}(51) & 161,8(11) & \mathrm{O}(1)-\mathrm{Cr}-\mathrm{O}(5) & 88,9(4) \\ \mathrm{O}(6)-\mathrm{Te}(2)-\mathrm{O}(31) & 95,6(4) & \mathrm{O}(1)-\mathrm{Cr}-\mathrm{O}(2) & 94,0(4) \\ \mathrm{O}(2)-\mathrm{Te}(2)-\mathrm{O}(6) & 95,6(2) & \mathrm{O}(1)-\mathrm{Cr}-\mathrm{O}(21) & 95,5(4) \\ \mathrm{O}(2)-\mathrm{Te}(2)-\mathrm{O}(31) & 93,0(4) & \mathrm{O}(3)-\mathrm{Cr}-\mathrm{O}(4) & 86,6(4) \\ \mathrm{O}(6)-\mathrm{Te}(2)-\mathrm{O}(31) & 95,6(3) & \mathrm{O}(3)-\mathrm{Cr}-\mathrm{O}(5) & 84,0(4) \\ \mathrm{O}(1)-\mathrm{Cr}-\mathrm{O}(3) & 172,8(4) & \mathrm{O}(3)-\mathrm{Cr}-\mathrm{O}(2) & 86,7(4) \\ \mathrm{O}(21)-\mathrm{Cr}-\mathrm{O}(4) & 87,2(4) & \mathrm{O}(3)-\mathrm{Cr}-\mathrm{O}(21) & 91,7(4) \\ \mathrm{O}(4)-\mathrm{Cr}-\mathrm{O}(5) & 89,8(4) & & \end{array}$

Une série de synthèses en tube d'or scellé a été réalisée à $700^{\circ} \mathrm{C}$ pour le système $\mathrm{Cr}_{2} \mathrm{O}_{3}-\mathrm{TeO}_{2}$ et $580^{\circ} \mathrm{C}$ pour le système $\mathrm{Al}_{2} \mathrm{O}_{3}-\mathrm{TeO}_{2}$. Ces températures devaient permettre une réactivité suffisamment élevée tout en nous préservant de la formation d'une éventuelle phase vitreuse. Dans ces conditions pas moins de quatre à cinq traitements thermiques entrecoupés de broyages soignés se sont avérés nécessaires pour obtenir une réaction totale. L'analyse radiocristallographique des divers échantillons polycristallins ainsi obtenus révèle que dans chacun des deux systèmes les spectres $\mathrm{X}$ des mélanges $\mathrm{M}_{2} \mathrm{O}_{3} .3 \mathrm{TeO}$ et $\mathrm{M}_{2} \mathrm{O}_{3} .4 \mathrm{TeO}$ sont identiques, ne révélant ni la présence dans le premier cas de $\mathrm{Cr}_{2} \mathrm{O}_{3}$ ou $\mathrm{Al}_{2} \mathrm{O}_{3}$ ni celle de $\mathrm{TeO}_{2}$ dans le deuxième cas.

De manière à confirmer les résultats structuraux nous avons eu recours à des mesures de densité par

Tableau 3. Coordonnées réduites et paramètres d'agitation thermique anisotrope $\left(\times 10^{4}\right)$

Les écarts types sont indiqués entre parenthèses.

\begin{tabular}{lcl} 
& Positions & \multicolumn{1}{c}{$x$} \\
$\mathrm{Te}(1)$ & $4(e)$ & $0,1397(1)$ \\
$\mathrm{Te}(2)$ & $4(e)$ & $0,6723(1)$ \\
$\mathrm{Cr}$ & $4(e)$ & $0,3192(3)$ \\
$\mathrm{O}(1)$ & $4(e)$ & $0,158(1)$ \\
$\mathrm{O}(2)$ & $4(e)$ & $0,563(1)$ \\
$\mathrm{O}(3)$ & $4(e)$ & $0,468(1)$ \\
$\mathrm{O}(4)$ & $4(e)$ & $0,127(1)$ \\
$\mathrm{O}(5)$ & $4(e)$ & $0,205(1)$ \\
$\mathrm{O}(6)$ & $2(d)$ & $\frac{1}{2}$
\end{tabular}

$y$
$0,8599(1)$
$0,8618(2)$
$0,5019(3)$
$0,649(1)$
$0,641(2)$
$0,375(1)$
$0,314(1)$
$0,624(1)$
0

\begin{tabular}{l}
\multicolumn{1}{c}{$z$} \\
$0,1762(1)$ \\
$0,4158(1)$ \\
$0,3842(2)$ \\
$0,4866(7)$ \\
$0,4517(8)$ \\
$0,2623(8)$ \\
$0,3429(9)$ \\
$0,2121(7)$ \\
$\frac{1}{2}$
\end{tabular}

$\beta_{11}$
$26(1)$
$92(2)$
$18(3)$
$49(14)$
$59(16)$
$25(14)$
$3(12)$
$59(15)$
$251(58)$

$\beta_{22}$
$21(1)$
$46(2)$
$22(3)$
$37(14)$
$57(16)$
$52(15)$
$29(14)$
$33(15)$
$121(40)$

$\beta_{33}$
$19(1)$
$18(1)$
$7(1)$
$16(6)$
$15(6)$
$15(6)$
$29(7)$
$4(5)$
$87(22)$

\begin{tabular}{cr}
$\beta_{12}$ & \multicolumn{1}{c}{$\beta_{13}$} \\
$1(1)$ & $0(1)$ \\
$34(1)$ & $-15(1)$ \\
$3(2)$ & $0(1)$ \\
$19(15)$ & $13(8)$ \\
$31(18)$ & $6(8)$ \\
$2(13)$ & $0(7)$ \\
$-7(9)$ & $-11(8)$ \\
$16(13)$ & $9(7)$ \\
$66(42)$ & $81(30)$
\end{tabular}

$\beta_{23}$
$2(1)$
$-10(1)$
$-2(1)$
$5(9)$
$8(12)$
$-9(9)$
$-11(8)$
$16(8)$
$-3(26)$


pycnométrie sur poudre dans l'orthophtalate de diéthyle ou le bromobenzène. Les résultats sont portés au Tableau 5. Les valeurs théoriques des densités ont été calculées sur la base de deux motifs (seule valeur possible) par maille monoclinique. Ces résultats confirment sans ambiguïté la formulation $\mathrm{M}_{2} \mathrm{Te}_{4} \mathrm{O}_{11}(\mathrm{M}=\mathrm{Al}, \mathrm{Cr})$ dérivée de l'étude structurale.

Tableau 5. Comparaison entre densités expérimentales et théoriques pour des échantillons de composition $\mathrm{M}_{2} \mathrm{Te}_{4} \mathrm{O}_{11}$ et $\mathrm{M}_{2} \mathrm{Te}_{3} \mathrm{O}_{9}$

$\begin{array}{ccc}\text { Composition de } & & \\ \text { l'échantillon } & d_{\text {exp }} & d_{\text {calc }}(Z=2) \\ \mathrm{Al}_{2} \mathrm{Te}_{4} \mathrm{O}_{11} & 5,02 \pm 0,05 & 5,06 \\ \mathrm{Al}_{2} \mathrm{Te}_{3} \mathrm{O}_{9} & 4,92 \pm 0,05 & 3,97 \\ \mathrm{Cr}_{2} \mathrm{Te}_{4} \mathrm{O}_{11} & 5,11 \pm 0,05 & 5,17 \\ \mathrm{Cr}_{2} \mathrm{Te}_{3} \mathrm{O}_{9} & 5,08 \pm 0,05 & 4,13\end{array}$

Récemment a été signalée dans le système $\mathrm{Fe}_{2} \mathrm{O}_{3}-$ $\mathrm{TeO}_{2}$, l'existence d'une phase de composition identique, $\mathrm{Fe}_{2} \mathrm{Te}_{4} \mathrm{O}_{11}$ dont la structure a été déterminée par Pertlik (1972). La maille est monoclinique, le groupe spatial $P 2_{1} / c$, les paramètres cristallins: $a=11,88, b=6,95$, $c=14,13 \AA, \beta=123,44^{\circ}(Z=4)$. La structure est cependant différente de celle de la phase $\mathrm{Cr}_{2} \mathrm{Te}_{4} \mathrm{O}_{11}$ que nous décrivons ici.

\section{Description de la structure et discussion}

Une projection de la structure sur la plan (010) est représentée à la Fig. 1. Les cotes $y$ des atomes qui y sont portées sont multipliées par 100 .

Chaque atome de chrome est en coordinence 6 , six atomes d'oxygène formant autour de lui un octaèdre légèrement distordu. $\mathrm{La}$ distance moyenne $\mathrm{Cr}-\mathrm{O}$ est égale à $1,974 \AA$, valeur en bon accord avec les données de la littérature et en particulier avec les deux valeurs 1,97 et $2,02 \AA$ trouvées pour les liaisons $\mathrm{Cr}-\mathrm{O}$ dans l'oxyde $\mathrm{Cr}_{2} \mathrm{O}_{3}$ (Newnham \& Haan, 1962). Ces octaèdres $\left(\mathrm{CrO}_{6}\right)$ sont liés deux à deux par l'intermédiaire d'une arête commune $\mathrm{O}(2)-\mathrm{O}(21)$, formant ainsi des anions complexes $\left(\mathrm{Cr}_{2} \mathrm{O}_{10}\right)^{14-}$. On peut remarquer d'ailleurs le raccourcissement classique de la distance entre atomes d'oxygène occupant les deux sommets de l'arête commune $[\mathrm{O}(2)-\mathrm{O}(21)=2,54 \AA]$. En corollaire les distances de l'atome de chrome à ces deux atomes d'oxygène $[\mathrm{Cr}-\mathrm{O}(2)=2,020$ et $\mathrm{Cr}-\mathrm{O}(21)=1,987 \AA]$ sont légèrement supérieures aux autres distances $\mathrm{Cr}-\mathrm{O}$ $(1,951,1,957,1,960$ et $1,970 \AA)$. Ces groupements $\left(\mathrm{Cr}_{2} \mathrm{O}_{10}\right)^{14-}$ se répètent toutes les demi-périodes le long de l'axe $O z$, alternativement en cote $y=0$ et $y=\frac{1}{2}$. Les axes $\mathrm{Cr}-\mathrm{Cr}$ de ces groupements sont tous parallèles.

Les atomes de tellure sont répartis sur deux types de sites cristallographiques notés $\mathrm{Te}(1)$ et $\mathrm{Te}(2)$.

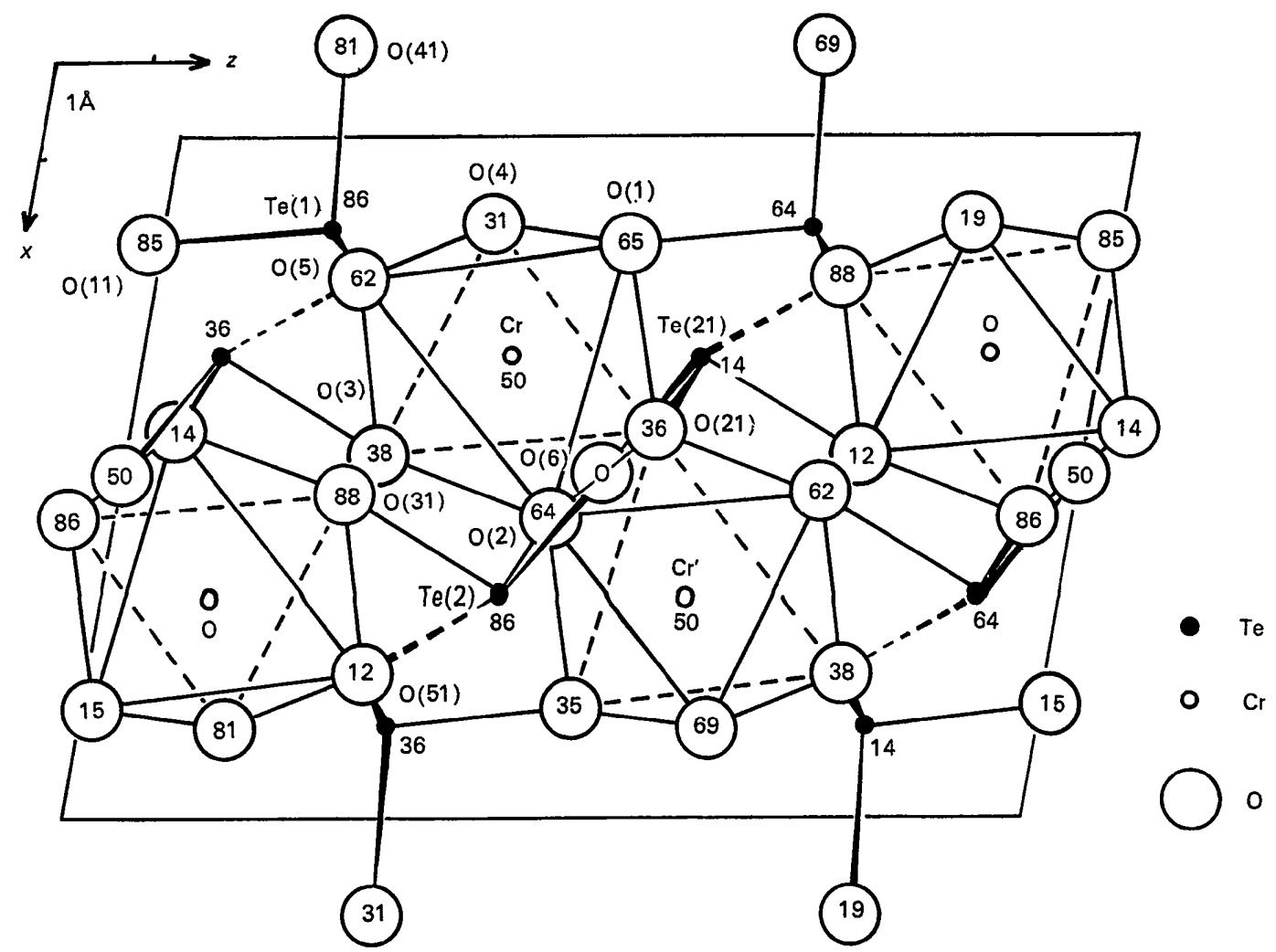

Fig. 1. Projection de la structure de $\mathrm{Cr}_{2} \mathrm{Te}_{4} \mathrm{O}_{11}$ sur le plan (010). 


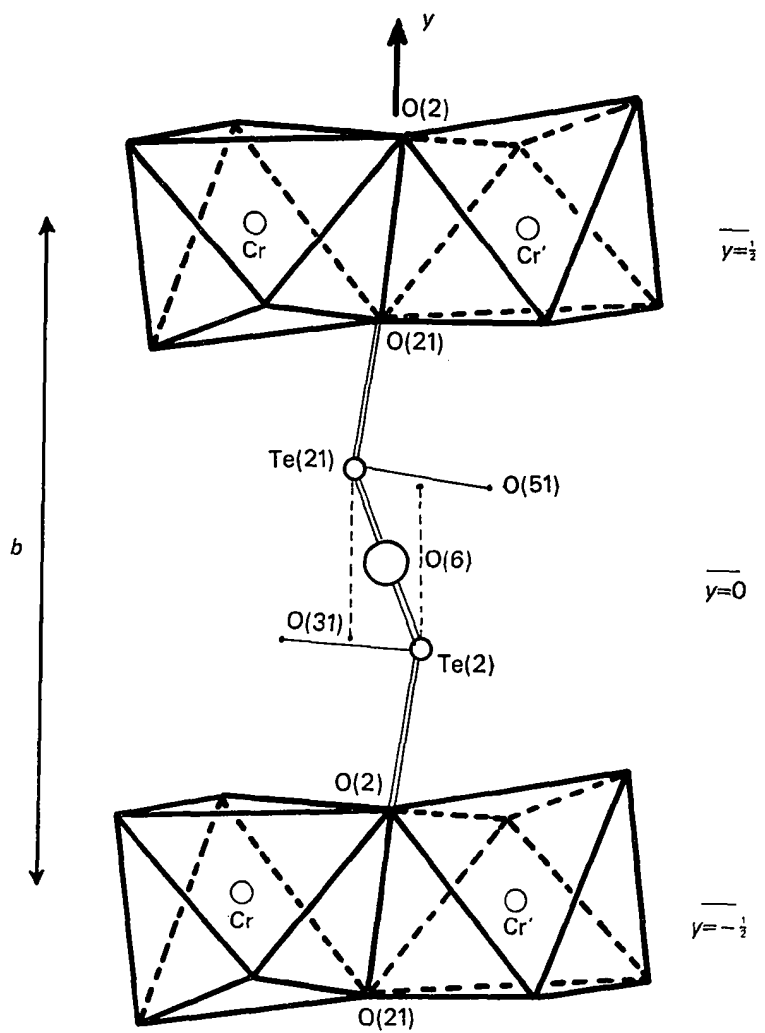

Fig. 2. Connexion de deux groupes $\left(\mathrm{Cr}_{2} \mathrm{O}_{10}\right)^{14-}$ par le groupe $\left(\mathrm{Te}_{2} \mathrm{O}\right)^{6+}$ selon l'axe $O y$.

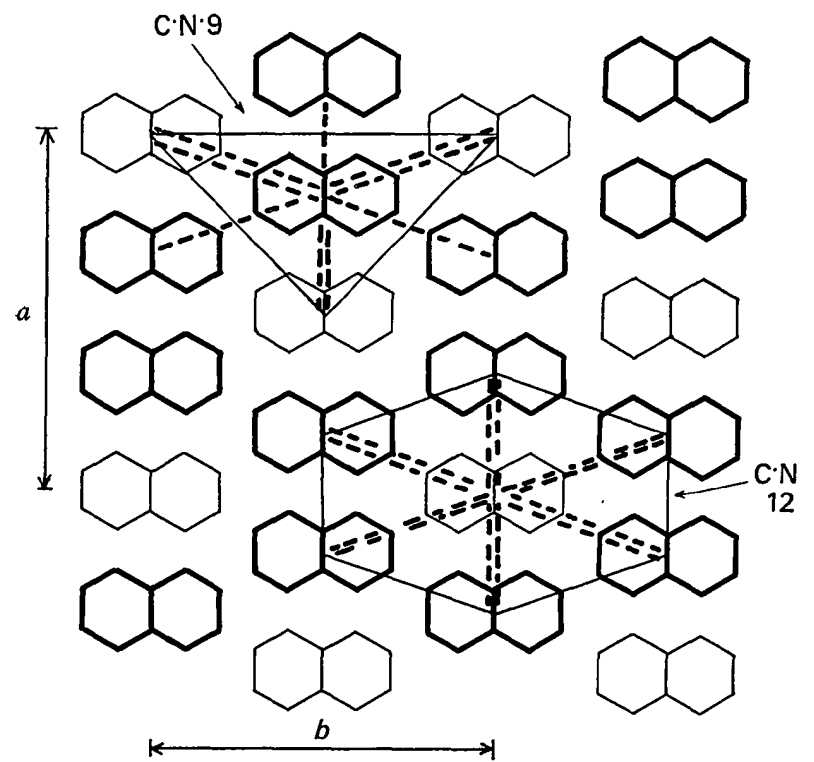

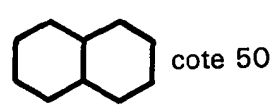

$\mathrm{Nb}_{2} \mathrm{Cl}_{10}$

Fig. 3. Schématisation de la structure de $\mathrm{NbCl}_{5}$.
En site $\mathrm{Te}(1)$ le tellure est lié à trois atomes d'oxygène avec lesquels il constitue une pyramide à base triangulaire $\left(\mathrm{TeO}_{3}\right)^{2-}$. Il possède en outre une paire non liée $E$, qui complète le polyèdre en tétraèdre $\left(\mathrm{TeO}_{3} E\right)^{2-}$. Les distances $\mathrm{Te}(1)-\mathrm{O}$ sont du même ordre que celles relevées dans les structures de $\mathrm{CuTeO}_{3} \cdot 2 \mathrm{H}_{2} \mathrm{O}$ (Zemann \& Zemann, 1962), $\mathrm{Te}_{2} \mathrm{~V}_{2} \mathrm{O}_{9}$ (Darriet \& Galy, 1973).

En site $\mathrm{Te}(2)$ le tellure présente une stéréochimie quelque peu différente, caractérisée par une coordinence $[3+1]$ déjà signalée par Pertlik \& Zemann (1971) et rencontrée notamment dans $\mathrm{TeVO}_{4} \alpha$ (Meunier et al., 1972).

Le quatrième atome d'oxygène $\mathrm{O}(51)$ étant relativement éloigné $[\mathrm{Te}(2)-\mathrm{O}(51)=2,562 \AA]$ nous ne considèrerons comme liés au tellure que les trois atomes d'oxygène: $O(2), O(6)$ et $O(31)$. En réalité les distances moyennes $\mathrm{Te}-\mathrm{O}$ pour les deux types d'atome de tellure en coordinence trois sont très voisines: $[\mathrm{Te}(1)-\mathrm{O}]$ moy. $=1,869 \AA$ et $[\mathrm{Te}(2)-\mathrm{O}] \mathrm{moy} .=1,874 \AA$. La différence essentielle entre les atomes $\mathrm{Te}(1)$ et $\mathrm{Te}(2)$ réside dans la formation pour les atomes $\mathrm{Te}(2)$ de groupements $\left[\mathrm{Te}(2)_{2} \mathrm{O}_{5}\right]^{2-}$ résultant de la mise en commun d'un atome d'oxygène $\mathrm{O}(6)$ par deux pyramides $\left[\mathrm{Te}(2) \mathrm{O}_{3}\right]^{2-}$. Il faut noter d'ailleurs le caractère très particulier de cet atome $\mathrm{O}(6)$ d'oxygène qui est le seul à ne pas être lié au chrome. Il n'est en effet lié qu'à deux atomes $\mathrm{Te}(2)$, l'angle $\mathrm{Te}(2)-\mathrm{O}(6)-\mathrm{Te}(2)$ valant exactement $180^{\circ}$. Ce groupement linéaire $\left(\mathrm{Te}_{2} \mathrm{O}\right)^{6+}$ constitue un exemple actuellement unique dans la cristallochimie du tellure $(+I V)$. De ce point de vue la formulation structurale de la phase $\mathrm{Cr}_{2} \mathrm{Te}_{4} \mathrm{O}_{11}$ peut s'écrire, tout au moins formellement: $\left[\mathrm{Cr}_{2} \mathrm{O}_{10}\right]^{14-}\left[\mathrm{Te}_{2} \mathrm{O}\right]^{6+}\left[\mathrm{Te}^{4+}\right]_{2}$, l'assemblage des anions complexes $\left(\mathrm{Cr}_{2} \mathrm{O}_{10}\right)^{14-}$ étant assuré par les atomes de tellure $\mathrm{Te}(1)$ et le groupement $\left[\mathrm{Te}(2)_{2} \mathrm{O}\right]^{6+}$ :

- l'atome $\mathrm{Te}(1)$ relie trois anions complexes, deux d'entre eux situés par exemple à la même cote moyenne 0 , le troisième situé alors à la cote moyenne $\frac{1}{2}$.

- le groupement $\left[\mathrm{Te}(2)_{2} \mathrm{O}\right]^{6+}$ en relie quatre, deux se succèdant avec une période $b$ le long de l'axe $O y$ et à la cote moyenne $\frac{1}{2}$, deux autres situés dans le plan $x O z$ et donc à la cote moyenne 0 (Fig. 2).

L'anion complexe $\left(\mathrm{Cr}_{2} \mathrm{O}_{10}\right)^{14-}$ peut être comparé à la molécule $\mathrm{Nb}_{2} \mathrm{Cl}_{10}$ caractéristique de la structure du pentachlorure de niobium $\mathrm{NbCl}_{5}$ (Talkin \& Sands, 1958). Dans cette structure également, deux octaèdres $\mathrm{NbCl}_{6}$ sont associés par une arête commune, formant la molécule $\mathrm{Nb}_{2} \mathrm{Cl}_{10}$. Un schéma de cette structure est donné à la Fig. 3. Les molécules sont en cote $z=0$ (traits fins) ou $z=\frac{1}{2}$ (traits épais). Dans l'empilement hexagonal compact que forment les atomes de chlore, les molécules $\mathrm{Nb}_{2} \mathrm{Cl}_{10}$ sont entourées soit par neuf molécules voisines [six d'entre elles formant un prisme trigonal, les trois autres étant situées à l'aplomb du centre de chaque face latérale (tri-capped trigonal prism)], soit par 12 molécules formant un prisme hexagonal. Il est intéressant dans le cas de la phase $\mathrm{Cr}_{2} \mathrm{Te}_{4} \mathrm{O}_{11}$, de considérer la disposition des anions complexes $\left(\mathrm{Cr}_{2} \mathrm{O}_{10}\right)^{14-}$; il apparaît que chacun d'eux est. 
entouré par six autres, formant un octaèdre aplati. Les distances de l'anion central $\left(\mathrm{Cr}_{2} \mathrm{O}_{10}\right)^{14-}$ aux six autres sont les suivantes:

- quatre distances équatoriales égales à $6,155 \AA$;

- deux distances axiales égales à $7,016 \AA$.

Une telle disposition est illustrée à la Fig. 4 par un schéma de la structure de $\mathrm{Cr}_{2} \mathrm{Te}_{4} \mathrm{O}_{11}$ où seuls les anions $\left(\mathrm{Cr}_{2} \mathrm{O}_{10}\right)^{14-}$ sont mis en évidence à l'aide d'un symbolisme analogue à celui déjà utilisé Fig. 3 pour représenter les molécules $\mathrm{Nb}_{2} \mathrm{Cl}_{10}$.

Si la coordinence de l'atome de tellure $\mathrm{Te}(1)$ ne présente pas de caractéristiques très originales, il faut par contre insister quelque peu sur le cas de l'atome $\mathrm{Te}(2)$. Nous avons vu que la coordinence finalement retenue pour cet atome est trois et que deux prismes $\left(\mathrm{TeO}_{3}\right)^{2-}$ sont unis par un sommet. Ils constituent ainsi un groupement $\left(\mathrm{Te}_{2} \mathrm{O}_{5}\right)^{2-}$ non seulement très rare dans la cristallochimie du tellure $(+\mathrm{IV})$, mais présentant de surcroît une configuration inédite. Il faut noter en effet que l'angle de liaison $\mathrm{Te}(2)-\mathrm{O}(6)-\mathrm{Te}(21)$ vaut très exactement $180^{\circ}$. Un tel groupement a été signalé seulement pour la deningite ( $\mathrm{Mn}, \mathrm{Ca}, \mathrm{Zn}$ ) $\mathrm{Te}_{2} \mathrm{O}_{5}$ étudiée par Walitzi (1961) et pour $\mathrm{Te}_{2} \mathrm{~V}_{2} \mathrm{O}_{9}$ dont la structure a été déterminée par Darriet \& Galy (1973), mais dans ces deux cas l'angle Te-O-Te est toujours inférieur à $180^{\circ}$, $128,8^{\circ}$ et $143,3^{\circ}$ respectivement. Notons également que lors d'études sur la cristallochimie du sélénium$(+\mathrm{IV})$, des groupements homologues $\left(\mathrm{Se}_{2} \mathrm{O}_{5}\right)^{2-}$ ont été mis en évidence dans les structures des phases $\mathrm{VSe}_{2} \mathrm{O}_{6}$ (Meunier, Bertaud \& Galy, 1974) et $\mathrm{ZnSe}_{2} \mathrm{O}_{5}$ (Meunier $\&$ Bertaud, 1974), mais que l'angle Se-O-Se a une valeur d'environ $120^{\circ}$.

\section{Références}

Cromer, D. T. \& W ABer, J. T. (1965). Acta Cryst. 18, 104 109.

Darriet, J. \& Galy, J. (1973). Cryst. Struct. Commun. 2, 237-238.

Frit, B. \& Hagenmuller, P. (1970). Bull. Soc. Chim. Fr. 7, 2491-2494.

Galy, J. \& Meunier, G. (1971). Acta Cryst. B27, 608-616.

Galy, J., Meunier, G., Andersson, S. \& Åström, A. (1975). J. Solid State Chem. 13, 142-159.

Meunier, G. (1974). Doctorat ès Sciences, Université de Bordeaux I.

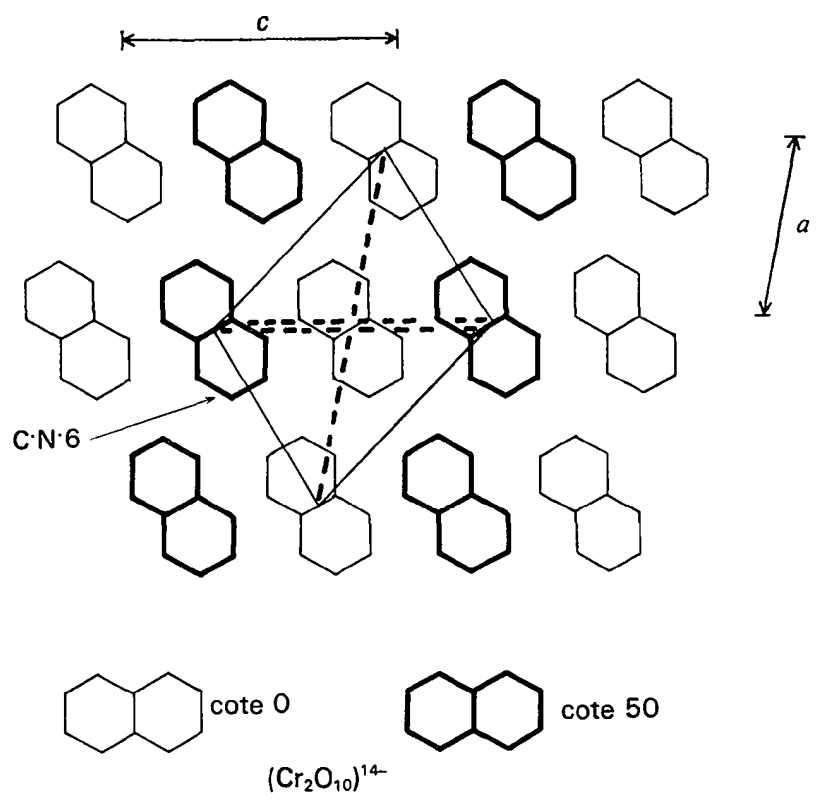

Fig. 4. Schématisation de l'arrangement tridimensionnel des anions $\left(\mathrm{Cr}_{2} \mathrm{O}_{10}\right)^{14-}$ dans la structure de $\mathrm{Cr}_{2} \mathrm{Te}_{4} \mathrm{O}_{11}$.

Meunier, G. \& Bertaud, M. (1974). Acta Cryst. B30, $2840-2843$.

Meunier, G., Bertaud, M. \& Galy, J. (1974). Acta Cryst. B30, 2834-2839.

Meunier, G., Darriet, J. \& Galy, J. (1972). J. Solid State Chem. 5, 314-320.

Meunier, G., Darriet, J. \& Galy, J. (1973). J. Solid State Chem. 6, 67-73.

Meunier, G. \& Galy, J. (1971). Acta Cryst. B27, 602-608. Meunier, G. \& Galy, J. (1973). Acta Cryst. B29, 12511255.

Newnham, R. E. \& HaAN, Y. M. (1962). Z. Kristallogr. 117, 235-241.

Pertlik, F. (1972). Mineral. Petrogr. Mitt. 18, 157-168.

Pertlik, F. \& ZemanN, J. (1971). Österr. Akad. Wiss. Math. Naturwiss. Kl. Anz. Jg. pp. 175-176.

Redman, M. J., Binnie, W. P. \& Mallio, W. J. (1971). J. Less.-Common Met. 23, 313-315.

Walitzi, E. M. (1961). Mineral. Petrogr. Mitt. 10, 241-249.

Zalkin, A. \& SANDS, D. E. (1958). Acta Cryst. 11, 615-622. Zemann, A. \& Zemann, J. (1962). Acta Cryst. 15, 698-710. 